\title{
Prevalence of Anxiety, Depression, and Post-Traumatic Stress Disorder Among Amputees Attending Jaipur Foot Trust Artificial Limb Centre in Kenya
}

Illham Mohamed

University of Nairobi

Teresia Mutavi ( $\sim$ Mutavi.teresia@uonbi.ac.ke)

University of Nairobi

Catherine Gitau

University of Nairobi

\section{Research Article}

Keywords: Amputees, Prevalence of Anxiety, Depression and PTSD

Posted Date: January 31st, 2022

DOI: https://doi.org/10.21203/rs.3.rs-1249771/v1

License: () (i) This work is licensed under a Creative Commons Attribution 4.0 International License. Read Full License 


\section{Abstract}

Background: Amputees have been noted to present with various psychiatric disorders including anxiety, body image disturbances, depression, and PostTraumatic Stress Disorder (PTSD). However, there is limited data available on the prevalence of anxiety, depression, and PTSD among amputees in Kenya despite the high incidences of amputations in Kenyan hospitals. This study aimed at finding out the prevalence of anxiety, depression, and post-traumatic stress disorder among amputees attending the Jaipur Foot Trust Center in Kenya.

Method: This study took a cross-sectional descriptive study design. One hundred and forty-one patients attending the Jaipur Foot Trust were recruited to participate in the study after giving informed consent using a non-probability purposive sampling method. A socio-demographic questionnaire was used to collect socio-demographic characteristics. The Patient Health Questionnaire (PHQ-9) was used to assess the patient's depression. The Generalized Anxiety Disorder (GAD-7) scale was used to assess the patient's anxiety level and The Impact of Event Scale (IES-R) was used to assess the severity of post-traumatic stress disorder (PTSD). Data analysis was done using Statistical Package for Social Science (SPSS) version 23.

Results: Findings from this study showed high rates of psychiatric morbidity where two-thirds of the patients reported PTSD (65\%) with more than threequarters of patients being diagnosed with depression (89.4\%) and anxiety (91.5\%). Also, there was a significant correlation between depression, anxiety, and PTSD, such that participants who had higher scores on anxiety and depression had significantly higher PTSD scores.

Conclusion: Anxiety, Depression, and PTSD are very common psychological reactions in patients who have undergone amputation. The researchers expected that some of the sociodemographic factors and some amputation-related characteristics would have a relation with psychiatric comorbidity. However, the findings of this study did not show any such relationship except the relation between anxiety, depression, and PTSD. Early psychological assessment and interventions after amputations will help alleviate psychological distress

\section{Introduction}

Amputations due to surgical procedural indications or traumatic events often result in a series of complex mental reactions in affected individuals. Most amputees go through these responses as an automatic attempt to cope with the situation but others present with debilitating psychiatric signs and symptoms[1]. Individuals who have gone through an amputation experience adverse psychological impacts that significantly affect their Quality of Life (QoL) [2]. Moreover, The World Health Organization (WHO) postulates that QoL can be influenced by factors relating to an individual's physical, cognitive, personal, social, spiritual, and environmental state [3].

Amputation has been associated with negative implications in three main ways namely: capacity, self-perception, and sensation. The anguish over the loss of sensation has been known to exacerbate self-consciousness that impacts one's functionality concerning sexuality and occupation often leading to social and psychosocial impairment [4]. It has been reported that traumatic limb loss is equivalent to other mainstream forms of loss such as bereavement, separation, divorce, castration anxiety as well as a fragmented sense of self, all of which summit to considerable cognitive disability and reduced quality of life [5].

The incapacitation of amputees to self and their families may in most cases cause psychiatric issues [5]. Among lower-limb amputees, factors such as being young, single, having low literacy levels, going through bilateral side amputation, experiencing trauma associated with the amputation, inability to get a prosthesis to aid in walking, shorter amputation duration, absence of medical comorbidity that could have necessitated the amputation, a wanting social support system and poor quality of life has been correlated to depression prevalence [6].

A study in a tertiary care hospital in Kashmir showed that in the cases of traumatic experiences resulting in severe amputations, Post-Traumatic Stress Disorder (PTSD) was at a distressing rate of $80 \%[7]$. These findings were replicated in other Western countries [8]. A study conducted in 41 countries attributed amputation and consequent PTSD from snakebites, these contributed to the burden of Disability Adjusted Life Years (DALYs) at 1.03million [9].

A cross-sectional study in 2019 aimed at understanding depression and associated features among 196 lower limb amputees aged 18-86 years [6]. The findings showed a $47.4 \%$ prevalence of depressive symptoms with $24.5 \%$ of them getting a Major Depressive Disorder. Another study on anxiety and depression among 56 lower limb amputees from both inpatient and outpatient hospitals and rehabilitation centers in Jordan showed that anxiety and depression were prevalent at $37 \%$ and $20 \%$ respectively[1]. Being female, poor social support, being unemployed having a traumatic amputation, recency of amputation, and below-knee amputation were found to be significantly related to higher psychological symptoms. Within this context, this study aimed to assess the prevalence of anxiety, depression, and PTSD among amputees attending the Jaipur Foot Trust Center in Nairobi, Kenya.

\section{Methodology}

The study adopted a descriptive cross-sectional design with a quantitative data collection approach. The study was done at the Jaipur Foot Trust artificial limb center located next to Kabete Barracks, along Waiyaki Way, Nairobi. It was established by the Rotary Club of Nairobi in the year 1990 to aid amputees to walk again. In this project, amputees are given artificial limbs at no cost. This service is endorsed by local donors that include individuals, groups, and institutions that are sympathetic to the objectives of the project. The population size of amputees that attended the Jaipur foot trust center was approximately 80 amputees per month translating to 960 amputees per year.

Ethical approval was sought from the University of Nairobi/Kenyatta National Hospital Ethics Review Committee [P678/12/2020]. The population consisted of all adult amputees attending the Jaipur Foot Trust. Those who refused to give consent were excluded. Using Cochran's formula [10] with an estimated prevalence of depression of $20 \%$ among amputees in India[4], the margin of error(precision) of $5 \%$ and a confidence interval of $95 \%$. The population of amputees attending Jaipur foot trusts center was approximately 80 amputees per month translating to 240 amputees over three months data collection 
period. After applying finite population correction the minimum sample size required was 122 participants. Allowing for a $10 \%$ non-response rate the minimum sample size required was 136 participants.

Participants were monitored for signs of respiratory disease and other primary distinguishing symptoms of COVID-19 disease, such as fever, cough, and shortness of breath or trouble breathing, as well as a history of recent exposure to individuals with COVID-19 disease, shortly before the face-to-face appointment. Participants with potential exposure or symptoms indicative of a respiratory condition were not invited for face-to-face visits. The researchers underwent regular temperature checks before entering the research site and correctly wore a facemask at all times during the face-to-face interactions. Suitable infection prevention control measures were ensured at the site of face-to-face visits, as follows: Temperature checks were carried out using a noncontact thermometer for all participants and other individuals arriving at the research site. There were hand-washing stations and hand sanitizers for all to use. During face-to-face interactions, the researchers ensured that participants correctly wore their face masks. A minimum physical distance of 1.5 meters in the waiting room was maintained.

Study participants were then recruited from patients receiving services at the Jaipur foot trust center using non probability purposive sampling technique. All eligible participants were recruited both new and those coming for checkups/follow-ups. The screening was done to assess whether the participants met the stated inclusion criteria. This process entailed giving an informed consent document with details of the study and the participants were allowed to ask questions they may have regarding the study. Participants who met the inclusion criteria and willing to participate in the study were requested to sign an informed consent form. They then proceeded to fill the Patient Health Questionnaire (PHQ-9), The Impact of Event Scale (IES-R), The Generalized Anxiety Disorder (GAD), and socio-demographic questionnaire.

THE PHQ-9 depression module scores each of the DSM-5 criteria as "Not at all” ("0") to "Nearly every day" (“3”) (K Kroenke, Spitzer, \& Williams, 2001). Therefore, the severity measure of the PHQ-9 ranges from 0-27 for depression where higher scores indicate high levels of depression. The depression severity tabulated according to the total score of every participant as a score of 0-4 no depression, 5-9 mild depression, 10-14 moderate depression, 15-19 moderately severe depression, and 20-27 severe depressive disorder.

The GAD-7 seven-item anxiety questionnaire uses a threshold score of 10 and has a sensitivity of $89 \%$ and specificity of $82 \%$ for GAD (Spitzer, Kroenke, Williams, \& Löwe, 2006). It has scores of "0" ("Not at all"), "1" ("Several days"), "2" ("More than half the days"), and "3" ("Nearly every day"). The scores of 5, 10 , and 15 are cut-off points from mild to severe anxiety where further evaluation is recommended for scores above 10.

The Impact of Event Scale (IES-R) is a DSM-5 self-report measure for assessing the subjective distress as a result of traumatic events (Weiss, 2007). The rating of items is on a 5-point scale ranging from " 0 " ("Not at all") to "4" ("Extremely") yielding a total score of 0 - 88. This total can be used to assess partial or full PTSD and has cut-off points for moderate and severe PTSD.

Descriptive statistics were used to scrutinize the general distribution of data and the depression and anxiety scores, using means and standard deviations for continuous variables and proportions for categorical variables. Independent samples t-test, One-way Analysis of Variance (ANOVA), was applied to identify group differences. Generalized linear models were used to categorize independent predictors of anxiety, depression, and PTSD. All analyses were conducted using Statistical Package for Social Science (SPSS) version 23. The statistical significance level was set at $p<0.05$ all tests will be 2 -tailed.

\section{Results}

As shown in table 1 below, the calculated sample size was 136, but 141 participants were interviewed because there was an increase in the number of respondents in the last few days and the researchers opted to assess willing respondents. Consequently, 141 questionnaires were analyzed reflecting 104 percent response rate. Age: The mean age was 43.4 years and ranged from 18-85 years, with the bulk of the participants aged between 31-50 years. Sex: More than half of the participants (55.3\%) were males and the rest (44.7\%) females. Marital Status: The majority of the participants were married (55.3\%), $31.9 \%$ were single and $12.9 \%$ were either divorced/separated/Widowed. Education level: In terms of education level $35.0 \%$ had completed secondary school, $29.3 \%$ had less than primary education, $20.7 \%$ had completed primary school and $15.0 \%$ had completed college/university education. Occupation: More than $2 / 3$ rds (69.3\%) were employed while $30.7 \%$ were unemployed. Monthly Income: In terms of monthly income, $45 \%$ had an income of $<20,000 \mathrm{Ksh}, 21.7 \%$ had an income of more than $20,000 \mathrm{Ksh}$ a month while the rest $33.3 \%$ had no income.

\section{Table 1: Socio-demographic Characteristics of the respondents}




\begin{tabular}{|c|c|c|c|}
\hline Variable & Category & Frequency $(\mathrm{N}=141)$ & Percentage (\%) \\
\hline \multirow[t]{2}{*}{ Sex } & Male & 78 & 55.3 \\
\hline & Female & 63 & 44.7 \\
\hline Age in Years & Mean; Median; Range & $43.4 ; 42 ; 18$ to 85 & \\
\hline \multirow[t]{5}{*}{ Age Category } & 30 and Below & 27 & 19.1 \\
\hline & $31-40$ Years & 35 & 24.8 \\
\hline & 41-50 Years & 39 & 27.7 \\
\hline & 51-60 Years & 20 & 14.2 \\
\hline & Above 60 & 20 & 14.2 \\
\hline \multirow[t]{3}{*}{ Marital Status } & Single & 45 & 31.9 \\
\hline & Married & 78 & 55.3 \\
\hline & Divorced/Separated/Widowed & 18 & 12.8 \\
\hline \multirow[t]{5}{*}{ Highest level of Education } & Less than Primary School & 41 & 29.3 \\
\hline & Primary School & 29 & 20.7 \\
\hline & Secondary/ High School & 49 & 35.0 \\
\hline & College/ University & 21 & 15.0 \\
\hline & Non-Response & 1 & \\
\hline \multirow[t]{3}{*}{ Employment Status } & Employed & 97 & 69.3 \\
\hline & Un-Employed & 43 & 30.7 \\
\hline & Non-Response & 1 & \\
\hline \multirow[t]{4}{*}{ Monthly Income } & No Income & 43 & 33.3 \\
\hline & $<20,000$ Ksh & 58 & 45.0 \\
\hline & 20,000 and Above & 28 & 21.7 \\
\hline & Non-Response & 12 & \\
\hline
\end{tabular}

\section{Psychosocial, Biological and other Characteristics of the Respondents}

As shown in table 2 below, the amputation type consisted of most (92.9\%) of the respondents had a unilateral amputation, while the rest had a bilateral amputation, of which $70 \%$ had their amputation below the knee. Causes of Amputation: Non-vasculitis causes accounted for the majority (80.7\%) of amputations of which included (56\%) were caused by road traffic accidents, followed by snake bites. Vasculitis causes accounted for $19.3 \%$ of the total amputation in which diabetes accounted for (70\%) and gangrene (30\%). Walking Aid: Prothesis was used by $73.6 \%$ of the respondents as a walking aid, $25 \%$ used crutches while $1.4 \%$ used a wheelchair. Presence of Other Illnesses: $23.4 \%$ of the respondents indicated that they have been diagnosed with other illnesses, of which $60.6 \%$ had diabetes, $45.5 \%$ had hypertension, $6.1 \%$ had asthma and arthritis respectively. Pain at the Amputation Site: $11.3 \%$ of the participants indicated that they experience pain at the amputation site which they rated at a scale of 1 to 10 . Social Support: The majority ( $87.9 \%$ ) of the respondents indicated that they receive social support from their families.

\section{Table 2: Psychosocial, Biological and other Characteristics of the Respondents}




\begin{tabular}{|c|c|c|c|}
\hline \multirow[t]{2}{*}{ Amputation Type } & Bilateral & 10 & 7.1 \\
\hline & Unilateral & 131 & 92.9 \\
\hline \multirow[t]{3}{*}{ Level of Amputation } & Above the Knee & 42 & 30.0 \\
\hline & Below Knee & 98 & 70.0 \\
\hline & Non-Response & 1 & \\
\hline \multirow[t]{3}{*}{ Reason for Amputation } & Non-Vasculitis & 113 & 80.7 \\
\hline & Vasculitis & 27 & 19.3 \\
\hline & Non-Response & 1 & \\
\hline \multirow[t]{4}{*}{ Type of walking Aid } & Wheel Chair & 2 & 1.4 \\
\hline & Prosthesis & 103 & 73.6 \\
\hline & Crutches & 35 & 25.0 \\
\hline & Non-Response & 1 & \\
\hline \multirow[t]{2}{*}{ Presence of other illness } & Yes & 33 & 23.4 \\
\hline & No & 108 & 76.6 \\
\hline \multirow[t]{2}{*}{ Experience pain at the amputation site } & Yes & 16 & 11.3 \\
\hline & No & 125 & 88.7 \\
\hline \multirow[t]{7}{*}{ Rate your pain on a scale of $1-10(\mathrm{~N}=16)$} & 2 & 4 & 25.0 \\
\hline & 3 & 3 & 18.8 \\
\hline & 4 & 1 & 6.3 \\
\hline & 5 & 3 & 18.8 \\
\hline & 6 & 3 & 18.8 \\
\hline & 7 & 1 & 6.3 \\
\hline & 9 & 1 & 6.3 \\
\hline \multirow[t]{2}{*}{ Receive Support from your family } & Yes & 124 & 87.9 \\
\hline & No & 17 & 12.1 \\
\hline
\end{tabular}

\section{Presence of other illness}

The respondents were requested to indicate the presence of other illnesses. From the figure 1 below, $60.6 \%$ indicated diabetes, $45.5 \%$ indicated hypertension, $18.2 \%$ indicated comorbidity, $6.1 \%$ indicated asthma while $6.1 \%$ indicated arthritis.

\section{Vasculitis amputation}

Further, the respondents were requested to indicate the vasculitis causes of amputation. As shown in the (figure 2) below, $70 \%$ of the respondents indicated diabetes while $30 \%$ of the respondents indicated gangrene.

\section{Non-Vasculitis amputation}

The respondents were requested to indicate the non-vasculitis causes of amputation. From the figure below (figure 3 ), $56 \%$ of the respondents indicated road traffic accidents, $14.3 \%$ indicated snake bites, $9.9 \%$ indicated physical injury, $8.8 \%$ indicated infections, $4.4 \%$ indicated gas explosion, $3.3 \%$ indicated congenital, $2.2 \%$ indicated cancer, $1.1 \%$ indicated boat accident while $1.1 \%$ indicated crocodile attack.

\section{Prevalence of PTSD, Depression, and Anxiety}

\section{Prevalence of PTSD}

A total of 92 participants screened positive for PTSD (Scores $\geq 33$ ) giving a prevalence rate of $65 \% 95 \%$ C.I. $57.4 \%$ to $73.0 \%$.while $35 \%$ of the respondents were negative for PTSD as shown in (table 3) below. This implies that most of the respondents were positive for PTSD. The Mean Median, SD, Min. Max and 
interquartile range are presented in Table 4.

\section{Prevalence of Depression}

As shown in Table 3, the prevalence of mild depression was $22.0 \%$ 95\% C.I. $15.6 \%$ to $29.1 \%$; Moderate depression $39.0 \% 95 \%$ C.I. $31.2 \%$ to $46.8 \%$; Moderately severe $27.0 \% 95 \%$ C.I. $19.9 \%$ to $34.0 \%$ and severe depression $1.4 \% 95 \%$ C.I. $0.0 \%$ to $3.5 \%$. (table 3 ). Among those who endorsed any item on the scale. The level of difficulty in carrying out the tasks was as follows $8.0 \%$ indicated that it was not difficult, $65.7 \%$ said it was somewhat difficult, $20.9 \%$ said it was very difficult and $6.0 \%$ said it was extremely difficult. The Mean Median, SD, Min. Max and interquartile range are presented in Table 4.

\section{Prevalence of Anxiety}

As shown in Table 3, the prevalence of mild anxiety was 30.5\% 95\% C.I. $23.4 \%$ to $38.3 \%$; moderate anxiety $40.4 \% 95 \%$ C.I. $32.6 \%$ to $48.9 \%$; and severe anxiety $20.6 \% 95 \%$ C.I. $13.5 \%$ to $27.7 \%$ (table 3 ). Among those who endorsed any item on the scale. The level of difficulty in carrying out the tasks was as follows $8.0 \%$ indicated that it was not difficult, $65.7 \%$ said it was somewhat difficult, $19.7 \%$ said it was very difficult and $6.6 \%$. The Mean Median, SD, Min. Max and interquartile range are presented in Table 4.

\section{Table 3: Prevalence of Depression Anxiety and PTSD}

\begin{tabular}{|c|c|c|c|c|c|}
\hline \multirow[t]{2}{*}{ Measure } & \multirow[t]{2}{*}{ Category } & \multirow{2}{*}{$\begin{array}{l}\text { Frequency } \\
(\mathrm{N}=141)\end{array}$} & \multirow{2}{*}{$\begin{array}{l}\text { Percentage } \\
(\%)\end{array}$} & \multicolumn{2}{|c|}{ 95\% C.I. } \\
\hline & & & & Lower & Upper \\
\hline \multirow[t]{2}{*}{ PTSD } & Negative for PTSD $(<33)$ & 49 & 34.8 & 27.0 & 42.6 \\
\hline & Positive for PTSD ( $\geq 33$ ) & 92 & 65.2 & 57.4 & 73.0 \\
\hline \multirow[t]{5}{*}{ Depression } & None (0-4) & 15 & 10.6 & 6.4 & 15.6 \\
\hline & Mild (5-9) & 31 & 22.0 & 15.6 & 29.1 \\
\hline & Moderate (10-14) & 55 & 39.0 & 31.2 & 46.8 \\
\hline & Moderately Severe (15-19) & 38 & 27.0 & 19.9 & 34.0 \\
\hline & Severe (20-27) & 2 & 1.4 & 0.0 & 3.5 \\
\hline \multirow[t]{4}{*}{ Anxiety } & Minimal Anxiety (0-4) & 12 & 8.5 & 4.3 & 13.5 \\
\hline & Mild Anxiety (5-9) & 43 & 30.5 & 23.4 & 38.3 \\
\hline & Moderate Anxiety (10-14) & 57 & 40.4 & 32.6 & 48.9 \\
\hline & Severe Anxiety (15-21) & 29 & 20.6 & 13.5 & 27.7 \\
\hline
\end{tabular}

Table 4: Descriptive Statistics of Outcome Measures

\begin{tabular}{|c|c|c|c|c|c|c|}
\hline \multirow[t]{3}{*}{ Measure } & \multirow{3}{*}{$\begin{array}{l}\text { Patient Health Questionnaire } \\
\text { PHQ-9 }\end{array}$} & \multirow{3}{*}{$\begin{array}{l}\text { General Anxiety Questionnaire } \\
\text { GAD }\end{array}$} & \multicolumn{4}{|c|}{$\begin{array}{l}\text { Impact of Events Scale-Revised } \\
\text { (IES-R) }\end{array}$} \\
\hline & & & IES-R & INT & AVD & HYP \\
\hline & & & \multicolumn{4}{|l|}{ Total } \\
\hline - Mean & 11.4 & 10.7 & 37.2 & 13.9 & 14.7 & 8.6 \\
\hline - Median & 12.0 & 11.0 & 37.0 & 14.0 & 14.0 & 9.0 \\
\hline \multicolumn{7}{|l|}{ - Std. Deviation } \\
\hline \multicolumn{7}{|l|}{ - Minimum } \\
\hline \multicolumn{7}{|l|}{ - Maximum } \\
\hline - Interquartile Ra & 7.0 & 6.0 & 17.5 & 8.0 & 8.0 & 5.0 \\
\hline
\end{tabular}

\section{Correlation between Depression, Anxiety, and PTSD}

Table 5: presents the Correlation between Depression, Anxiety, and PTSD. The Correlation between PTSD and Depression scores was r=0.688; $<<0.001 ;$ PTSD and Anxiety scores were $r=0.759 ; p<0.001$, Anxiety and Depression scores were $r=0.719 ; p<0.001$. 
Table 5: Correlation between Depression, Anxiety and PTSD

\begin{tabular}{lllllll} 
Pearson Correlation & 1 & 2 & 3 & 4 & 5 & 6 \\
\hline 1. PTSD & 1 & & & & & \\
\hline 2. Depression & $0.688^{* *}$ & 1 & & & & \\
\hline 3. Anxiety & $0.759^{* *}$ & $0.719^{* *}$ & 1 & & & \\
\hline 4. INT-IES-R subscale & $0.955^{* *}$ & $0.743^{* *}$ & $0.756^{* *}$ & 1 & & \\
\hline 5. AVD-IES-R subscale & $0.928^{* *}$ & $0.529^{* *}$ & $0.670^{* *}$ & $0.817^{* *}$ & 1 & \\
\hline 6. HYP-IES-R subscale & $0.894^{* *}$ & $0.647^{* *}$ & $0.681^{* *}$ & $0.822^{* *}$ & $0.730^{* *}$ & 1
\end{tabular}

Note: **Correlation is significant at the 0.01 level (2-tailed).

Socio-demographic and Other Factors Associated with Depression

Table 6: presents the socio-demographic and other factors associated with depression (Bivariate analysis). Participants who were employed had significantly higher depression scores as compared to those who were unemployed $(\mathbf{p}=\mathbf{0 . 0 2 5})$. Respondents who had unilateral amputation had significantly higher depression scores as compared to those who had bilateral $(\mathbf{p}=\mathbf{0 . 0 2 2})$.

Table 6: Socio-demographic and Other Factors Associated with Depression ( Bivariate Analysis) 


\begin{tabular}{|c|c|c|c|c|}
\hline Variable & Category & $\mathbf{N}$ & Mean (SD) & p-Value \\
\hline \multirow[t]{2}{*}{$\operatorname{Sex}^{\dagger}$} & Male & 78 & $10.9(5.1)$ & \multirow[t]{2}{*}{0.195} \\
\hline & Female & 63 & $12.0(4.6)$ & \\
\hline \multirow[t]{5}{*}{ Age Category $\ddagger$} & 30 and Below & 27 & $10.1(6.5)$ & \multirow[t]{5}{*}{0.140} \\
\hline & $31-40$ Years & 35 & $12.3(4.4)$ & \\
\hline & 41-50 Years & 39 & $12.0(4.2)$ & \\
\hline & $51-60$ Years & 20 & $12.0(4.2)$ & \\
\hline & Above 60 & 20 & $9.6(4.7)$ & \\
\hline \multirow[t]{3}{*}{ Marital Status ${ }^{\ddagger}$} & Single & 45 & $11.5(5.7)$ & \multirow[t]{3}{*}{0.670} \\
\hline & Married & 78 & $11.1(4.5)$ & \\
\hline & Divorced/Separated/Widowed & 18 & $12.2(4.3)$ & \\
\hline \multirow[t]{4}{*}{ Highest level of Education ${ }^{\ddagger}$} & Less than Primary School & 41 & $11.4(4.3)$ & \multirow[t]{4}{*}{0.578} \\
\hline & Primary School & 29 & $10.4(4.4)$ & \\
\hline & Secondary/ High School & 49 & $11.6(5.6)$ & \\
\hline & College/ University & 21 & $12.3(5.1)$ & \\
\hline \multirow[t]{2}{*}{ Employment Status $^{\dagger}$} & Employed & 97 & $12.0(4.7)$ & \multirow[t]{2}{*}{0.025} \\
\hline & Un-Employed & 43 & $10.0(5.2)$ & \\
\hline \multirow[t]{3}{*}{ Monthly Income } & No Income & 43 & $10.0(5.2)$ & \multirow[t]{3}{*}{0.061} \\
\hline & $<20,000$ Ksh & 58 & $11.6(5.1)$ & \\
\hline & 20,000 and Above & 28 & $12.8(4.1)$ & \\
\hline \multirow[t]{2}{*}{ Amputation Type ${ }^{\dagger}$} & Bilateral & 10 & $8.0(7.5)$ & \multirow[t]{2}{*}{0.022} \\
\hline & Unilateral & 131 & $11.6(4.6)$ & \\
\hline \multirow[t]{2}{*}{ Level of Amputation ${ }^{\dagger}$} & Above the Knee & 42 & $12.1(4.7)$ & \multirow[t]{2}{*}{0.254} \\
\hline & Below Knee & 98 & $11.1(5.0)$ & \\
\hline \multirow[t]{2}{*}{ Reason for Amputation ${ }^{\dagger}$} & Non-Vasculitis & 113 & $11.4(5.2)$ & \multirow[t]{2}{*}{0.980} \\
\hline & Vasculitis & 27 & $11.4(3.5)$ & \\
\hline \multirow[t]{2}{*}{ Underlying illness $^{\dagger}$} & Yes & 33 & 11.7(3.4) & \multirow[t]{2}{*}{0.681} \\
\hline & No & 108 & $11.3(5.3)$ & \\
\hline \multirow[t]{2}{*}{ Experience pain at the amputation site ${ }^{\dagger}$} & Yes & 17 & $11.5(4.8)$ & \multirow[t]{2}{*}{0.505} \\
\hline & No & 124 & $10.6(5.6)$ & \\
\hline
\end{tabular}

Note: $\mathbf{t - I n d e p e n d e n t ~ s a m p l e s ~ t - t e s t ; ~ ¥ - O n e ~ w a y ~ a n a l y s i s ~ o f ~ v a r i a n c e - A N O V A ~}$

Socio-demographic and Other Factors Associated with Anxiety

Table 7: presents the socio-demographic and other factors associated with anxiety (Bivariate analysis). Female participants had significantly higher anxiety scores as compared to males $(\mathbf{p}=\mathbf{0 . 0 2 4})$.

Table 7: Socio-demographic and Other Factors Associated with Anxiety 


\begin{tabular}{|c|c|c|c|c|}
\hline Variable & Category & $\mathbf{N}$ & Mean (SD) & p-Value \\
\hline \multirow[t]{2}{*}{$\operatorname{Sex}^{+}$} & Male & 78 & $10.0(4.5)$ & \multirow[t]{2}{*}{0.024} \\
\hline & Female & 63 & $11.6(3.9)$ & \\
\hline \multirow[t]{5}{*}{ Age Category $¥$} & 30 and Below & 27 & $10.4(5.9)$ & \multirow[t]{5}{*}{0.957} \\
\hline & $31-40$ Years & 35 & $11.1(4.3)$ & \\
\hline & 41-50 Years & 39 & 10.7(3.4) & \\
\hline & $51-60$ Years & 20 & $10.9(4.1)$ & \\
\hline & Above 60 & 20 & $10.2(4.1)$ & \\
\hline \multirow[t]{3}{*}{ Marital Status ${ }^{\ddagger}$} & Single & 45 & $10.9(5.5)$ & \multirow[t]{3}{*}{0.823} \\
\hline & Married & 78 & $10.5(3.7)$ & \\
\hline & Divorced/Separated/Widowed & 18 & 11.1(3.9) & \\
\hline \multirow[t]{4}{*}{ Highest level of Education $¥$} & Less than Primary School & 41 & $10.7(4.1)$ & \multirow[t]{4}{*}{0.915} \\
\hline & Primary School & 29 & $10.2(3.9)$ & \\
\hline & Secondary/ High School & 49 & $10.9(4.5)$ & \\
\hline & College/ University & 21 & $11.0(5.3)$ & \\
\hline \multirow[t]{2}{*}{ Employment Status $^{\dagger}$} & Employed & 97 & $11.0(4.0)$ & \multirow[t]{2}{*}{0.154} \\
\hline & Un-Employed & 43 & $9.9(4.9)$ & \\
\hline \multirow[t]{3}{*}{ Monthly Income $e^{\ddagger}$} & No Income & 43 & $9.9(4.9)$ & \multirow[t]{3}{*}{0.340} \\
\hline & $<20,000 K s h$ & 58 & $11.1(3.8)$ & \\
\hline & 20,000 and Above & 28 & $11.2(4.8)$ & \\
\hline \multirow[t]{2}{*}{ Amputation Type ${ }^{\dagger}$} & Bilateral & 10 & $8.3(6.4)$ & \multirow[t]{2}{*}{0.068} \\
\hline & Unilateral & 131 & $10.9(4.1)$ & \\
\hline \multirow[t]{2}{*}{ Level of Amputation ${ }^{\dagger}$} & Above the Knee & 42 & $11.0(4.2)$ & \multirow[t]{2}{*}{0.641} \\
\hline & Below Knee & 98 & $10.7(4.4)$ & \\
\hline \multirow[t]{2}{*}{ Reason for Amputation ${ }^{\dagger}$} & Non-Vasculitis & 113 & $11.4(5.2)$ & \multirow[t]{2}{*}{0.980} \\
\hline & Vasculitis & 27 & 11.4(3.5) & \\
\hline \multirow[t]{2}{*}{ Underlying illness $^{\dagger}$} & Yes & 33 & $11.0(2.7)$ & \multirow[t]{2}{*}{0.694} \\
\hline & No & 108 & $10.6(4.7)$ & \\
\hline \multirow[t]{2}{*}{ Experience pain at the amputation site ${ }^{\dagger}$} & Yes & 17 & $10.7(4.3)$ & \multirow[t]{2}{*}{0.908} \\
\hline & No & 124 & $10.8(4.7)$ & \\
\hline
\end{tabular}

Note: t-Independent samples t-test; ¥-One way analysis of variance-ANOVA

\section{Socio-demographic and Other Factors Associated with PTSD}

Table 8 presents the socio-demographic and other factors associated with PTSD (Bivariate analysis). Respondents who had unilateral amputation had significantly higher PTSD scores as compared to those who had bilateral $(\mathbf{p}=\mathbf{0 . 0 3 4})$.

Table 8: Socio-demographic and Other Factors Associated with PTSD 


\begin{tabular}{|c|c|c|c|c|}
\hline Variable & Category & $\mathbf{N}$ & Mean (SD) & p-Value \\
\hline \multirow[t]{2}{*}{$\operatorname{Sex}^{+}$} & Male & 78 & 36.2(15.) & \multirow[t]{2}{*}{0.348} \\
\hline & Female & 63 & 38.5(13.) & \\
\hline \multirow[t]{5}{*}{ Age Category $\ddagger$} & 30 and Below & 27 & $38.1(21)$. & \multirow[t]{5}{*}{0.962} \\
\hline & 31-40 Years & 35 & 36.8(13.) & \\
\hline & 41-50 Years & 39 & $36.8(11)$. & \\
\hline & 51-60 Years & 20 & $38.9(9.4)$ & \\
\hline & Above 60 & 20 & $36.0(13)$. & \\
\hline \multirow[t]{3}{*}{ Marital Status ${ }^{\ddagger}$} & Single & 45 & $36.8(18)$. & \multirow[t]{3}{*}{0.822} \\
\hline & Married & 78 & 37.0(12.) & \\
\hline & Divorced/Separated/Widowed & 18 & $39.2(8.9)$ & \\
\hline \multirow[t]{4}{*}{ Highest level of Education ${ }^{\ddagger}$} & Less than Primary School & 41 & 34.6(13.) & \multirow[t]{4}{*}{0.306} \\
\hline & Primary School & 29 & 36.1(15.) & \\
\hline & Secondary/ High School & 49 & 38.1(13.) & \\
\hline & College/ University & 21 & 41.5(16.) & \\
\hline \multirow[t]{2}{*}{ Employment Status $^{\dagger}$} & Employed & 97 & 37.5(12.) & \multirow[t]{2}{*}{0.647} \\
\hline & Un-Employed & 43 & 36.3(17.) & \\
\hline \multirow[t]{3}{*}{ Monthly Income $e^{\ddagger}$} & No Income & 43 & 36.3(17.) & \multirow[t]{3}{*}{0.554} \\
\hline & $<20,000 K s h$ & 58 & 36.5(13.) & \\
\hline & 20,000 and Above & 28 & 39.9(13.) & \\
\hline \multirow[t]{2}{*}{ Amputation Type ${ }^{\dagger}$} & Bilateral & 10 & 28.1(22.) & \multirow[t]{2}{*}{0.034} \\
\hline & Unilateral & 131 & 37.9(13.) & \\
\hline \multirow[t]{2}{*}{ Level of Amputation ${ }^{\dagger}$} & Above the Knee & 42 & $36.9(14)$. & \multirow[t]{2}{*}{0.840} \\
\hline & Below Knee & 98 & 37.5(14.) & \\
\hline \multirow[t]{2}{*}{ Reason for Amputation ${ }^{\dagger}$} & Non-Vasculitis & 113 & $11.4(5.2)$ & \multirow[t]{2}{*}{0.980} \\
\hline & Vasculitis & 27 & $11.4(3.5)$ & \\
\hline \multirow[t]{2}{*}{ Underlying illness $^{\dagger}$} & Yes & 33 & 38.4(11.) & \multirow[t]{2}{*}{0.576} \\
\hline & No & 108 & $36.8(14)$. & \\
\hline \multirow[t]{2}{*}{ Experience pain at the amputation site ${ }^{\dagger}$} & Yes & 17 & $36.8(13)$. & \multirow[t]{2}{*}{0.378} \\
\hline & No & 124 & 40.1(16.) & \\
\hline
\end{tabular}

Note: t-Independent samples t-test; $¥-$ One way analysis of variance-ANOVA

\section{Independent Predictors of Depression (multivariate analysis)}

Table 9: presents the independent predictors of depression after adjusting for all other variables that were associated with anxiety at the bivariate level. Participants who were employed had significantly higher depression scores as compared to those who were unemployed ( $\beta=1.33 ; 95 \%$ C.I. 0.16 to 2.49 ; $p=0.030)$. Participants who had higher scores of anxiety and PTSD had significantly higher depression scores $(\beta=-0.50 ; 95 \%$ C.I. 0.31 to $0.69 ; p<0.001)$ and $(\beta=-0.12 ; 95 \%$ C.I. 0.06 to $0.17 ; p<0.001)$ respectively.

\section{Table 9: Independent Predictors of Depression (multivariate analysis)}




\begin{tabular}{|c|c|c|c|c|c|c|}
\hline \multirow[t]{2}{*}{ Variable } & \multirow[t]{2}{*}{ Category } & \multirow[t]{2}{*}{$\beta$} & \multirow[t]{2}{*}{ S.E. } & \multicolumn{2}{|c|}{ 95\% Confidence Interval } & \multirow[t]{2}{*}{ Sig. } \\
\hline & & & & Lower & Upper & \\
\hline \multirow[t]{2}{*}{ Gender } & Male & 0.32 & 0.56 & -0.78 & 1.41 & 0.57 \\
\hline & Female & Ref. & & & & \\
\hline \multirow[t]{2}{*}{ Amputation Type } & Bilateral & -1.13 & 1.05 & -3.19 & 0.94 & 0.28 \\
\hline & Unilateral & Ref. & & & & \\
\hline \multirow[t]{2}{*}{ Employment status } & Employed & 1.33 & 0.59 & 0.16 & 2.49 & 0.030 \\
\hline & Unemployed & Ref. & & & & \\
\hline Anxiety & & 0.50 & 0.10 & 0.31 & 0.69 & $<0.001$ \\
\hline PTSD & & 0.12 & 0.03 & 0.06 & 0.17 & $<0.001$ \\
\hline
\end{tabular}

\section{Independent Predictors of PTSD (multivariate analysis)}

Table 10: presents the independent predictors of depression after adjusting for all other variables that were associated with PTSD at the bivariate level. Participants who had higher scores of anxiety and depression had significantly higher PTSD scores $(\beta=-1.81 ; 95 \%$ C.I. 1.32 to $2.29 ; p<0.001)$ and $(\beta=-0.87 ; 95 \%$ C.I. 0.44 to $1.30 ; p<0.001)$ respectively.

\section{Table 10: Independent Predictors of PTSD (multivariate analysis)}

\begin{tabular}{|c|c|c|c|c|c|c|}
\hline \multirow[t]{2}{*}{ Variable } & \multirow[t]{2}{*}{ Category } & \multirow[t]{2}{*}{$\beta$} & \multirow[t]{2}{*}{ S.E. } & \multicolumn{2}{|c|}{ 95\% Confidence Interval } & \multirow[t]{2}{*}{ Sig. } \\
\hline & & & & Lower & Upper & \\
\hline \multirow[t]{2}{*}{ Gender } & Male & 1.29 & 1.53 & -1.71 & 4.29 & 0.400 \\
\hline & Female & Ref. & & & & \\
\hline \multirow[t]{2}{*}{ Amputation Type } & Bilateral & -2.37 & 2.89 & -8.03 & 3.29 & 0.412 \\
\hline & Unilateral & Ref. & & & & \\
\hline \multirow[t]{2}{*}{ Employment status } & Employed & -2.37 & 1.65 & -5.60 & 0.86 & 0.150 \\
\hline & Unemployed & Ref. & & & & \\
\hline Anxiety & & 1.81 & 0.25 & 1.32 & 2.29 & $<0.001$ \\
\hline Depression & & 0.87 & 0.22 & 0.44 & 1.30 & $<0.001$ \\
\hline
\end{tabular}

Note: †-Independent samples t-test; †-One way analysis of variance-ANOVA

\section{Discussion}

Amputations are surgeries that mutilate and also disrupt the patients' everyday lives. These procedures are also considered distasteful. Moreover, most of them are necessitated in developing countries following trauma and diseases, while amputations in more developed countries are considered for trauma, diabetes, and peripheral vascular disease $[11,12]$. Findings from this study revealed that middle-aged men (mean age of 43.4 years) among the study participants have undergone amputation. This can be compared to a similar study where the population had a mean age of 43.8 years [13]. However, this differs from most of the other studies, where most of the study participants were of the younger age group [14,15]. Most of the participants were male in this study. This is similar to another study done in Ireland [16]. Nevertheless, most studies have shown little disparity in regards to the clinical results of men and women in terms of psychological well-being following amputation.

At the bivariate level, female participants had significantly higher anxiety scores as compared to males at ( $p=0.024)$. Similarly, a study done in Jordan showed that forty-four percent of females had anxiety compared to thirty-six percent of males[1]. Non-vasculitis causes accounted for the majority (80.7\%) of amputations. Trauma was postulated to be the common cause of amputations in more than $50 \%$ of cases, with a significant percentage being as a result of road traffic accidents as earlier reported [17]. This may be used to explain why anxiety, depression, and Post Traumatic Stress Disorder (PTSD) were reported to be higher in this population than in others $[14,5]$.

Our study found that while adjusting for gender and amputation type, employment was a risk factor for depression and was also highly associated with anxiety and PTSD. We included factors such as gender, amputation type, and employment status in our final model due to the reported increased risk of loss of functionality for male participants as they may be breadwinners for their families, and thus amputations significantly affect their employability based on their severity [18]. It is also consistent that people who experience amputation-related motor skills loss tend to make them more susceptible to adverse reactions [19]. Most of the participants reported stigma at the workplace that affected their emotional well-being. In this study, no association was reported between the demographic parameters such as age, marital status, income, level of amputation, the reason for amputation, pain at the amputation 
site, and depression. Similarly, another study [20], did not find a correlation between age, gender, level of amputation and, etiology of amputation with development of psychiatric morbidity. On the contrary, a study in Malaysia found that factors such as being young, single, having low literacy levels, going through bilateral side amputation, experiencing trauma associated with the amputation, inability to get a prosthesis to aid in walking, shorter amputation duration, absence of medical comorbidity that could have necessitated the amputation, a wanting social support system and poor quality of life were correlated to the prevalence of depression [6].

The findings of this study revealed that a high proportion of individuals who undergo amputation suffered from Anxiety, Depression, and PTSD. The observed rates of psychiatric morbidity where two-thirds of the patients reported PTSD with more than three-quarters of patients being diagnosed with depression and anxiety is alarming. This finding is not distant from what other previous studies have found where researchers reported depression as a highly prevalent psychiatric comorbid condition in amputees, ranging between $13 \%$ and $32 \%$. Depression prevalence among amputees in Mexico has also been reported to be as high as $92.5 \%$ [14] which is comparable to what has been found in this study though the sample size was smaller compared to this study by 40 . Amputees may present with depressive symptoms [21,22]. Moreover, It has been shown that the presence of depressive symptoms may be linked to an array of debilitating outcomes like increased pain intensity, restriction of activity, self-consciousness, body image associated anxiety, and a significantly reduced quality of life [23, 24]. Some studies propose that between $15 \%$ and $26 \%$ of persons with limb loss might experience PTSD [22]. In another study from Kashmir Valley, researchers reported the occurrence of psychiatric comorbidity in people with traumatic amputation from PTSD prevalence to be at $20 \%$ and $80 \%$, respectively [25]. The high prevalence is similar to what was found in this study since trauma accounted for more than fifty percent of the reason for the amputations. The causes for the elevated PTSD prevalence can be related to the amputation itself, or the incident that lead to the amputation, or a mixture of both factors [7]. Moreover, there was a significant correlation between depression, anxiety, and PTSD, such that participants who had higher scores on anxiety and depression had significantly higher PTSD scores and vice versa. A study done to provide conclusive information concerning the psychological distress among amputees in India established that a considerable number of people who undergo an amputation tend to develop psychological distress and psychiatric disorders [5].

\section{Conclusion}

In conclusion, depression, anxiety, and PTSD are very common psychological reactions in patients who have undergone amputation. We expected that some of the sociodemographic factors and some amputation-related characteristics would have had a relation with psychiatric comorbidity, but the findings of this study did not show any such relationship except relation between PTSD, depression and anxiety.There is a need to lay down hospital policies that screen for mental illness in patients undergoing amputations. Early psychological assessment and interventions after amputations will help prevent psychological illnesses. Given the high level of depression, anxiety, and PTSD among amputees, the surgical treatment providers need to liaise with psychiatrists and psychologists so that a comprehensive psychological evaluation can be done when required, and treatment of psychiatric disorders if identified can be initiated. Hence necessary steps to identify and manage psychiatric illness in amputees be initiated in clinical settings. Implementation of stringent road safety regulations would be a feasible control measure. Employers need to make work-related adjustments for employees with disabilities such as accessible lifts and ramps.

\section{Strengths And Limitations Of The Study}

The strengths of the study include a relatively large number of amputees, and the use of a structured interview scale adds to the study's strengths. There are few important limitations of the current study that need to be mentioned and addressed in future studies, i.e., poor representation of the female gender, short duration of amputation history, and lack of control group (nontraumatic amputation cases).

Data collection took place amid the covid-19 pandemic, which has greatly contributed to the high prevalence rate. The pandemic has significant social, economic, and cultural impacts on people's lives. Measures that were taken to combat the pandemic affected day-to-day activities. Containment measures like lockdowns that were imposed by the government, to mitigate covid-19 spread were not conducive to production and processing industries and hence some of the workers were dismissed, [26]. For the majority of amputees, transport fees had to be sent through mobile money transfer to facilitate the fixing of the prosthesis.

\section{Abbreviations}

1. PTSD Post Traumatic Stress Disorder

2. GAD-7 Generalized Anxiety Disorder

3. IES-R Impact of Event Scale Revised

4. SPSS Statistical Package for Social Science

5. DALYs Disability Adjusted Life Years

6. ANOVA One-way Analysis of Variance

7. WHO World Health Organization

8. QoL Quality of Life

\section{Declarations}

Ethical approval was sought from the University of Nairobi/Kenyatta National Hospital Ethics Review Committee [P678/12/2020]. Informed consent from the participants was sought before administering the questionnaires.

Page $12 / 15$ 


\section{References}

1. Hawamdeh, Z. M., Othman, Y. S., \& Ibrahim, A. I. (2008). Assessment of anxiety and depression after lower limb amputation in Jordanian patients. Neuropsychiatric disease and treatment, 4(3), 627.

2. O'Donnell, M. L., Creamer, M., Elliott, P., Atkin, C., \& Kossmann, T. (2005). Determinants of quality of life and role-related disability after injury: impact of acute psychological responses. Journal of Trauma and Acute Care Surgery, 59(6), 1328-1335.

3. Whoqol Group. (1995). The World Health Organization quality of life assessment (WHOQOL): position paper from the World Health Organization. Social science \& medicine, 41(10), 1403-1409. (1995). The World Health Organization quality of life assessment (WHOQOL): Position paper from the World Health Organization. Social Science and Medicine, 41(10), 1403-1409. https://doi.org/10.1016/0277-9536(95)00112-K

4. Baby, S., Chaudhury, S., \& Walia, T. S. (2018). Evaluation of treatment of psychiatric morbidity among limb amputees. Industrial psychiatry journal, 27(2), 240.

5. Sahu, A., Gupta, R., Sagar, S., Kumar, M., \& Sagar, R. (2017). A study of psychiatric comorbidity after traumatic limb amputation: A neglected entity. Industrial Psychiatry Journal, 26(2), 228. https://doi.org/10.4103/ipj.ipj_80_16

6. Iqbal, M., Mohamed, S., \& Mohamad, M. (2019). Depression and Its Associated Factors among Lower Limb Amputees at Hospital Kuala Lumpur and Hospital Sultanah Bahiyah: A Cross-Sectional Study.

7. Margoob, Mushtaq \& Khan, Akash \& Gani, Naseem \& Maajid, Ab \& Mansur, Imtiyaz \& Bhat, Mohammed \& Mushtaq, Huda \& Jeelani, Hinan \& Nehra, D. (2008). P. of P. T. S. D. after A. A. P. S. from K. J. practitioner: a journal of current clinical medicine \& surgery. 15. 5-7. (n.d.). (PDF) Prevalence of Post Traumatic Stress Disorder after Amputation: A Preliminary Study from Kashmir. Retrieved August 9,

2020,fromhttps://www.researchgate.net/publication/256199925_Prevalence_of_Post_Traumatic_Stress_Disorder_after_Amputation_A_Preliminary_Study_

8. Desmond, D., Gallagher, P., Henderson-Slater, D., \& Chatfield, R. (2008). Pain and psychosocial adjustment to lower limb amputation amongst prosthesis users. Prosthetics and Orthotics International, 32(2), 244-252. https://doi.org/10.1080/03093640802067046

9. Halilu, S., Iliyasu, G., Hamza, M., Chippaux, J. P., Kuznik, A., \& Habib, A. G. (2019). Snakebite burden in Sub-Saharan Africa: estimates from 41 countries. Toxicon, 159, 1-4. https://doi.org/10.1016/j.toxicon.2018.12.002

10. Cochran, W. G. (1977). Sampling Techniques. New York: John Wiley \& Sons, Inc.

11. Mosaku, K. S., Akinyoola, A. L., Fatoye, F. O., \& Adegbehingbe, O. O. (2009a). Psychological reactions to amputation in a sample of Nigerian amputees. General Hospital Psychiatry, 31(1), 20-24https://doi.org/10.1016/j.genhosppsych.2008.08.004

12. Onuminya, J. E., Obekpa, P. O., Ihezue, H. C., Ukegbu, N. D., \& Onabowale, B. O. (2000). Major amputations in Nigeria: A plea to educate traditional bonesetters. Tropical Doctor, 30(3), 133-135. https://doi.org/10.1177/004947550003000306

13. Falgares, G., Lo Gioco, A., Verrocchio, M. C., \& Marchetti, D. (2019). Anxiety and depression among adult amputees: the role of attachment insecurity, coping strategies, and social support. Psychology, Health \& Medicine, 24(3), 281-293.https://doi.org/10.1080/13548506.2018.1529324

14. Vázquez, P. I. A., Castillo Avila, R. G., Del Dominguez Zentella, M. C., Hernández-Díaz, Y., González-Castro, T. B., Tovilla-Zárate, C. A., ... Frésan, A. (2018). Prevalence and correlations between suicide attempt, depression, substance use, and functionality among patients with limb amputations. International Journal of Rehabilitation Research, 41(1), 52-56. https://doi.org/10.1097/MRR.0000000000000259

15. Padovani, M. T., Martins, M. R. I., Venâncio, A., \& Forni, J. E. N. (2015). Anxiety, depression and quality of life in individuals with phantom limb pain. Acta Ortopedica Brasileira, 23(2), 107-110. https://doi.org/10.1590/1413-78522015230200990

16. Gallagher, P., O'Donovan, M. A., Doyle, A., \& Desmond, D. (2011). Environmental barriers, activity limitations, and participation restrictions experienced by people with major limb amputation. Prosthetics and Orthotics International, 35(3), 278-284. https://doi.org/10.1177/0309364611407108

17. Ogeng'O, J. A., Obimbo, M. M., \& King'Ori, J. (2009). A pattern of limb amputation in a Kenyan rural hospital. International Orthopaedics, 33(5), 14491453. https://doi.org/10.1007/s00264-009-0810-5

18. Washington, J. (2013). The Relations among Psychological and Demographic Factors in Individuals with Lower Limb Amputation. Senior Theses and Projects. Retrieved from https://digitalrepository.trincoll.edu/theses/337

19. (2018). Emotional and Psychological Reactions to Amputation - Physiopedia. Retrieved January 29, 2020, from Physiopedia website: https://www.physiopedia.com/Emotional_and_Psychological_Reactions_to_Amputation

20. Singh, R., Ripley, D., Pentland, B., Todd, I., Hunter, J., Hutton, L., \& Philip, A. (2009). Depression and anxiety symptoms after lower limb amputation: The rise and fall. Clinical Rehabilitation, 23(3), 281-286. https://doi.org/10.1177/0269215508094710

21. Cavanagh, S. R., Shin, L. M., Karamouz, N., \& Rauch, S. L. (2006). Psychiatric and emotional sequelae of surgical amputation. Psychosomatics, 47(6), 459-464. https://doi.org/10.1176/appi.psy.47.6.459

22. Phelps, L. F., Williams, R. M., Raichle, K. A., Turner, A. P., \& Ehde, D. M. (2008). The Importance of Cognitive Processing to Adjustment in the 1 st Year Following Amputation. Rehabilitation Psychology, 53(1), 28-38. https://doi.org/10.1037/0090-5550.53.1.28

23. Asano, M., Rushton, P., Miller, W. C., \& Deathe, B. A. (2008). Predictors of quality of life among individuals who have a lower limb amputation. Prosthetics and Orthotics International, 32(2), 231-243. https://doi.org/10.1080/03093640802024955

24. Hanley, M. A., Jensen, M. P., Ehde, D. M., Hoffman, A. J., Patterson, D. R., \& Robinson, L. R. (2004). Psychosocial predictors of long-term adjustment to lower-limb amputation and phantom limb pain. Disability and Rehabilitation, 26(14-15), 882-893. https://doi.org/10.1080/09638280410001708896

25. Muzaffar, N., Mansoor, I., Hafeez, A., \& Margoob, M. (2012). Psychiatric comorbidity in amputees with average sociodemographic status and the role of theologic and family support in a conflict zone. Australasian Journal of Disaster and Trauma Studies, 2012(1), 31-38.

26. Suleiman, M. A. (2020). Assessing the Economic Security Impacts of Covid-19 Pandemic: Key Responses and Challenges in Kenya. 
Figures

Figure 1

See image above for figure legend

\section{Vasculitis Casue of Amputation}

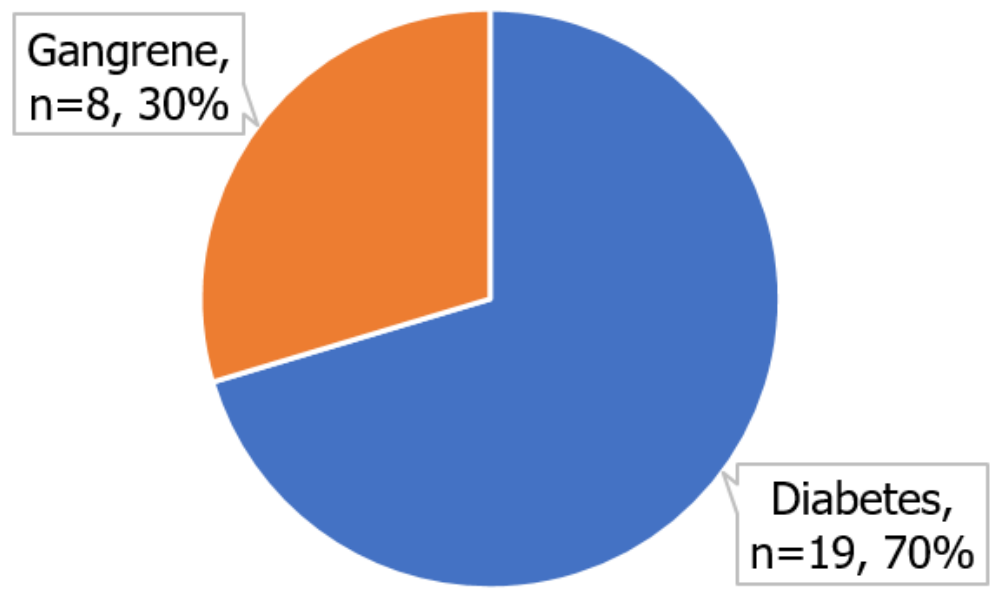

Figure 2

See image above for figure legend

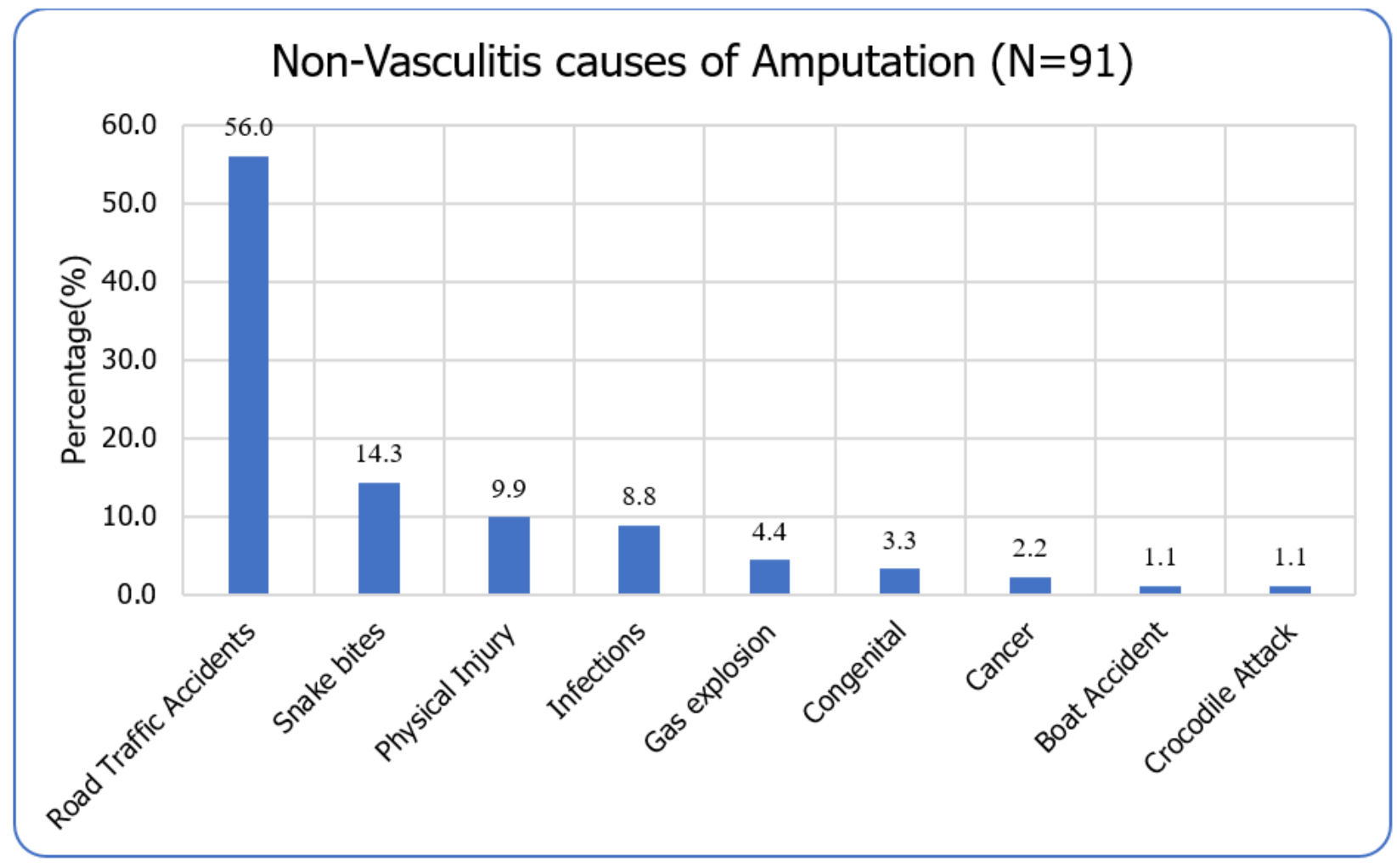

Figure 3 
See image above for figure legend

Page 15/15 